\title{
Partial core vaporization during Giant Impacts inferred from the entropy and the critical point of iron
}

\author{
${\text { Zhi } \text { Li }^{\mathrm{a}, *} \text {, Razvan Caracas }}^{\mathrm{a}, \mathrm{b}, *}$, François Soubiran ${ }^{\mathrm{a}}$ \\ a CNRS, Ecole Normale Supérieure de Lyon, Laboratoire de Géologie de Lyon UMR 5276, Centre Blaise Pascal, 46 allée d'Italie, 69364 Lyon, France \\ b The Center for Earth Evolution and Dynamics (CEED), University of Oslo, Oslo, Norway
}

\section{A R T I C L E I N F O}

\section{Article history:}

Received 10 February 2020

Received in revised form 2 July 2020

Accepted 5 July 2020

Available online 22 July 2020

Editor: F. Moynier

\section{Keywords:}

Giant Impacts

core vaporisation

entropy

critical point

iron

\begin{abstract}
A B S T R A C T
Giant impacts are disruptive events occurring in the early stages of planetary evolution. They may result in the formation of a protolunar disk or of a synestia. A central planet and one or several moons condense upon cooling bearing the chemical signature of the silicate mantles of the initial bodies; the iron cores may partly vaporize, fragment and/or merge. Here we determine from ab initio simulations the critical point of iron in the temperature range of $9000-9350 \mathrm{~K}$, and the density range of $1.85-2.40 \mathrm{~g} / \mathrm{cm}^{3}$, corresponding to a pressure range of 4-7 kbars. This implies that the iron core of the proto-Earth may become supercritical after giant impacts and during the condensation and cooling of the protolunar disk. We show that the iron core of Theia partially vaporized during the Giant Impact. Part of this vapor may have remained in the disk, to eventually participate in the Moon's small core. Similarly, during the late veneer a large fraction of the planetesimals have their cores undergoing partial vaporization. This would help mixing the highly siderophile elements into magma ponds or oceans.
\end{abstract}

(C) 2020 The Author(s). Published by Elsevier B.V. This is an open access article under the CC BY license (http://creativecommons.org/licenses/by/4.0/).

\section{Introduction}

Understanding the behavior of iron during extreme shock is critical to correctly model planetary cores during disruptive impacts. Once differentiated, planets and planetesimals cores are dominated by liquid or solid iron, alloyed with nickel and various lighter elements (Hirose et al., 2013). Because of its obvious geophysical significance, considerable effort was put to determine both theoretically and experimentally its phase diagram (Alfè et al., 1999; Campbell, 2016; Caracas, 2016; Tateno et al., 2010) up to Earth's inner core conditions (around $360 \mathrm{GPa}$ and $6000 \mathrm{~K}$ ) and beyond. Pressures of $1400 \mathrm{GPa}$ were reached using high power lasers at the National Ignition Facility (Smith et al., 2018). Recently, a complete set of equations of state (EOS) was proposed, covering 7$30 \mathrm{~g} / \mathrm{cm}^{3}$ densities and 10,000-1,000,000 K temperatures (Sjostrom and Crockett, 2018).

The studies of iron at low density are scarce. The density of liquid iron at 0.2 GPa has been measured by Hixson et al. (1990) up to $4000 \mathrm{~K}$. Grosse and Kirshenbaum (1963) measured the liquidvapor equilibrium density up to the boiling point at 1 bar and

\footnotetext{
* Corresponding authors at: CNRS, Ecole Normale Supérieure de Lyon, Laboratoire de Géologie de Lyon UMR 5276, Centre Blaise Pascal, 46 allée d'Italie, 69364 Lyon, France.

E-mail addresses: zhi.li@ens-lyon.fr (Z. Li), razvan.caracas@ens-lyon.fr (R. Caracas).
}

$3160 \mathrm{~K}$. In order to obtain the critical point, a large extrapolation must be made. The first method is to employ empirical equations of state with several parameters, which can be determined from available experimental data (Fortov and Lomonosov, 2010; Medvedev, 2014); the second one is to use the law of rectilinear diameter (Grosse and Kirshenbaum, 1963). However, it is unclear whether these extrapolations work at high temperature where no experimental data are available. Indeed, the regime of low densities and high temperatures, which is still not yet well characterized, is typical for the after-shock state of proto-planetary cores occurring in the aftermath of catastrophic events such as giant impacts.

The Earth's Moon formed after such a giant impact between the proto-Earth and Theia, an astronomical body whose most commonly accepted size is that of Mars (Asphaug, 2014; Canup, 2004a). Hydrodynamic impact simulations show that it results in the formation of a disk (Canup, 2012; Canup and Righter, 2000; Cuk and Stewart, 2012) or a synestia (Lock et al., 2018). The disk might be iron-depleted, producing a small or even non-existent Moon core. However, results of these simulations heavily rely on available EOS. An experimental result on iron found the shock pressure required for vaporization when compressed from ambient conditions and then decompressed to 1 bar to be around $507(+65$, -85) GPa (Kraus et al., 2015), lower than previous estimates of 887 GPa (Pierazzo et al., 1997). A lowering of the vaporization threshold pressure makes impactors with even lower velocities prone to 
releasing iron vapor from their core. This implies that the cores of a large number of the planetesimals from the late stages of accretion largely vaporized during the impacts (Kraus et al., 2015).

In order to assess whether the core of the planets undergoes significant vaporization during a giant impact, we employ ab initio molecular-dynamics simulations, to explore iron over a wide density region encompassing the critical point (CP) and the Hugoniot lines of the shocked cores. As the liquid-vapor dome ends at $\mathrm{CP}$, the position of the latter determines the time evolution of the protolunar disk/synestia during its condensation. The regions in the disk that lie outside the liquid-vapor dome, above $\mathrm{CP}$ in the supercritical state and/or in the liquid stability field, are formed of one homogeneous fluid phase. Vapor and liquid phases separate below $\mathrm{CP}$, resulting in chemical segregation and different mixing and convection regimes.

\section{Calculation details}

\subsection{First-principles molecular-dynamics calculations}

We study the hot fluid iron at temperatures extending into the supercritical state using first-principles (FP) molecular-dynamics (MD) simulations. In MD simulations, the particles follow the Newtonian dynamics under the action of interatomic forces. We perform NVT simulations, where the number of particles, $N=108$ atoms, and the volume, $\mathrm{V}$, is kept fixed; the temperature, $\mathrm{T}$, is allowed to fluctuate around a constant average value using the Nosé thermostat (Nosé, 1984). We use a timestep of 1 fs. The total simulation time at each temperature and density condition is at least 10 ps. The interatomic forces are computed using the density-functional theory in the VASP implementation (Kresse and Furthmüller, 1996). We employ the projector-augmented wavefunction (PAW) flavor of the DFT (Blöchl, 1994; Kresse and Joubert, 1999), using the Perdew-Burke-Ernzerhof formalism (Perdew et al., 1996) of the generalized-gradient approximation for the exchange correlation term. We consider an iron pseudopotential with eight valence electrons $\left(3 d^{7} 4 s^{1}\right)$. We sample the Brillouin zone in the gamma point. The energy cut-off for the plane-wave basis set was set to $550 \mathrm{eV}$. The number of electronic bands was adapted to the density and temperature conditions such as to cover the entire spectrum of the fully and partially occupied states and to include enough non-occupied bands. The convergence of the pressure tensor and the energy are on the order of a few percent when compared to a grid of $4 \times 4 \times 4 \mathrm{k}$-points at a kinetic energy cut-off of $850 \mathrm{eV}$. Additional tests using 324 atoms show little effect on the pressure of the fluid iron.

It should be noted that both experiments (Waseda and Suzuki, 1970) and theoretical simulations (Lichtenstein et al., 2001) suggest that liquid iron is in a paramagnetic state. As discussed by Marqués et al. (2015), the spin-polarized MD simulations yield a small, but inherent and fluctuating residual long-range ferromagnetic order. In order to avoid such residual magnetic state, we decided to perform non-spin-polarized simulations to approximate the paramagnetic state of liquid iron at low pressure and high temperature. This is the mean field approximation of the paramagnetic state, even if it neglects the spin fluctuations.

\subsection{Construction of the spinodal and determination of the critical point}

During the simulations at low temperatures, with decreasing density the pressure reaches a local minimum. This marks the liquid spinodal point, the minimal density at which the liquid is stable: at densities lower than the spinodal, the liquid is unstable and cavitation occurs (Speedy, 1982). Under further expansion, the pressure starts to increase; the local maximum marks the gas spinodal - the maximum density at which the gas is metastable.
Between the gas and liquid spinodal densities, neither gas nor liquid can exist as a single phase, but rather they co-exist. This is similar to the van der Waals gas-liquid equilibrium model. In order to fit the pressure - density curves we employ a simple thirdorder polynomial function, as this polynomial approximates well the van der Waals relation. This method has been successfully used by other theoretical studies on supercooled silicon (Vasisht et al., 2011). Spinodal lines with negative pressure have been reported in experiments (Green et al., 1990), classic MD simulations on the metastable extension of liquid water (Poole et al., 1992), and firstprinciples MD on the metastable extension of liquid silicon (Zhao et al., 2016).

\subsection{Entropy calculations}

The release after shock is done along quasi-isentropic trajectories. Hence knowing its value along the Hugoniot equation of state of shocks allows us to reconstruct the entropic state of the protolunar disk. We can determine the entropy for a fluid in several steps. The starting point is the atomic velocity autocorrelation function, defined as:

$\Phi(t)=\frac{\left\langle\sum_{i=1}^{N} v_{i}(0) v_{i}(t)\right\rangle}{\left\langle\sum_{i=1}^{N} v_{i}(0) v_{i}(0)\right\rangle}$,

where $v_{i}$ is the atomic velocity of the $i$ th atom, \langle\rangle represents the ensemble average and $N$ is the total number of atoms. The Fouriertransform of the velocity auto-correlation function yields the total movement of the atoms in the fluid, defined as,

$F(v)=\int_{0}^{\infty} \Phi(t) \cos (2 \pi v t) d t$

The entropy can then be obtained by integrating over the vibrational part of this spectrum, in the same way as we do for solids. However, Equation (2) captures not only the agitation of the atoms but also their diffusion. The latter is zero in solids, which allows us to directly obtain the entropy; but for fluids by definition it is finite and positive, and thus must be removed from the spectrum of the Equation (2).

For this we employ the two-phase thermodynamic method (Lin et al., 2003) to decompose the total spectrum of Equation (2) into a diffusive, gas-like part and a purely vibrational solid-like part:

$F(v)=\left(1-f_{g}\right) F_{S}(v)+f_{g} F_{S}(v)$,

where $f_{g}$ is the gas-like fraction. The entropy stemming from the gas-like and the solid-like parts is obtained using the hard sphere model and the harmonic oscillator model respectively. This method gives a reasonable estimation of entropy for pure liquid metals (Desjarlais, 2013). We verify again our implementation and use the same parameters as Desjarlais (2013) and conduct simulations at 0 $\mathrm{GPa}$ and $1800 \mathrm{~K}$ for liquid iron. We obtain a value for the entropy of $11.05 \mathrm{k}_{\mathrm{B}}$ /atom, compared to $12.00 \mathrm{k}_{\mathrm{B}}$ /atom in Desjarlais (2013). The discrepancy comes from the magnetic entropy, which is estimated to be about $1 \mathrm{k}_{\mathrm{B}}$ /atom (Desjarlais, 2013) and which we did not include in our calculation.

\subsection{Transport properties}

The asymptotic slope of the mean square displacement with respect to time yields the diffusion coefficient $D$ in the long-time limit:

$D=\frac{1}{6} \lim _{\tau \rightarrow \infty} \frac{\operatorname{MSD}(\tau)}{\tau}$. 
Here, the atomic mean square displacement (MSD) is defined as the average \langle\rangle of the square of the distance traveled by the atoms $i$ in a period of time $\tau$, as:

$\operatorname{MSD}(\tau)=\left\langle\left[r_{i}\left(t_{0}+\tau\right)-r_{i}\left(t_{0}\right)\right]^{2}\right\rangle$.

The time origin $t_{0}$ is arbitrary. $\tau$ represents a sliding time window spanning a portion of the trajectory. The values are averaged over the total number of atoms and time origins.

\section{Results and discussion}

\subsection{Ab initio simulations to find the critical point}

We perform first-principles molecular dynamics simulations in the 3000-15000 K temperature range and densities below $8 \mathrm{~g} / \mathrm{cm}^{3}$. This regime is characteristic of the aftermath conditions of giant impacts (Canup, 2004b). We compute the pressure dependence of the density along several isotherms (Fig. 1). As simulations of the low-density gas phase may have inherent shortcomings related to ergodicity (Reed and Flurchick, 1994), we focus on the liquid spinodal, which lies at accessible densities.

Our simulations give at $3000 \mathrm{~K}$ and ambient pressure conditions a liquid density of $6.2 \mathrm{~g} / \mathrm{cm}^{3}$, in excellent agreement with experimental values of $6.22 \mathrm{~g} / \mathrm{cm}^{3}$ (Hixson et al., 1990). At the same pressure but $4000 \mathrm{~K}$, the liquid density decreases to $5.7 \mathrm{~g} / \mathrm{cm}^{3}$. This is only $0.2 \mathrm{~g} / \mathrm{cm}^{3}$ larger than the experimental value of 5.5 $\mathrm{g} / \mathrm{cm}^{3}$ obtained from experimental measurements (Hixson et al., 1990). This good agreement indicates the reliability of our ab initio methodology.

We use a third-order polynomial expansion of the pressure as a function of density to identify the liquid spinodal and the position of the critical point, as detailed in the methodology. For iron, we identify a liquid spinodal point for all isotherms up to $9000 \mathrm{~K}$. Along this latter isotherm the minimum pressure corresponding to the liquid spinodal is obtained at $2.40 \mathrm{~g} / \mathrm{cm}^{3}$. At $9000 \mathrm{~K}$, we extend the simulations towards even lower densities, which allows us to observe also a maximum along the pressure - density curve. This corresponds to the gas spinodal, lying at $1.85 \mathrm{~g} / \mathrm{cm}^{3}$. Starting with the $9350 \mathrm{~K}$ isotherm the pressure varies monotonically without any local minimum or maximum; this is characteristic of the supercritical state. Therefore, the position of the $\mathrm{CP}$ is bracketed by the two spinodal lines, which intersect in the CP itself, and by the last isotherm with minima and maxima and the first isotherm with monotonical pressure variation. For iron, using the results of our simulations we predict that the CP lies in the $1.85-2.40 \mathrm{~g} / \mathrm{cm}^{3}$, and $9000-9350 \mathrm{~K}$ range (Fig. 1). These values correspond to pressure of 4-7 kbars.

\subsection{Conditions for vaporization}

The behavior of materials under shock can be described using the Rankine-Hugoniot equations. These equations relate the density, pressure, and internal energy after shock to the initial state by,

$E-E_{0}=\frac{\left(P+P_{0}\right)\left(V_{0}-V\right)}{2}$,

where $E, P, V$ are the internal energy, pressure and volume, respectively. And the 0 subscript denotes the initial state. The MD simulations that we performed at various isotherms contain all the information needed to build the Hugoniot EOS.

We consider two representative initial states. The first case has iron at $1 \mathrm{GPa}$ and $1500 \mathrm{~K}$, conditions similar to what we could expect to have in small planetesimals. For these conditions that we call warm Hugoniot the EOS intercepts the iron melting curve

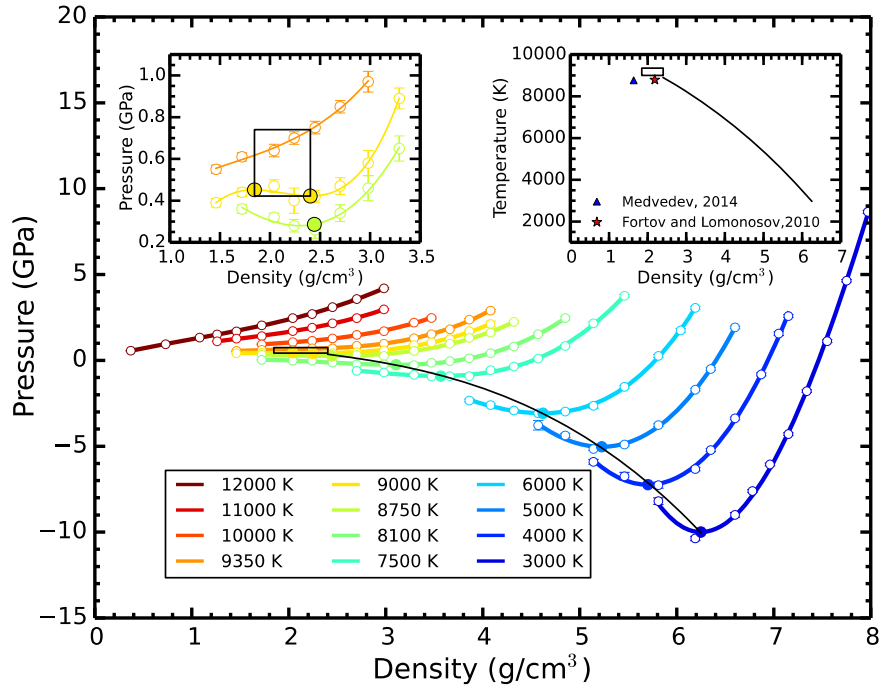

Fig. 1. Variation of pressure as a function of density for iron along several isotherms. Along a given isotherm below the critical temperature, with volume expansion, the pressure may decrease to reach negative values. These negative pressures indicate the presence of hydrostatic tension in the system. According to the classic nucleation theory (Karthika et al., 2016), the first-order transitions need to overcome energy barriers due to the surface energy, which prevents the formation of the thermodynamic stable phase. Therefore, this stage is thermodynamically metastable but mechanically stable. The minimum of the pressure marks the liquid spinodal (solid symbols). Joining the spinodal points yield the spinodal line (the black solid line). At densities lower than that of the liquid spinodal the pressure starts to increase until it reaches a maximum, which marks the gas spinodal, as shown in the left inset figure. At densities between the two spinodal lines a two-phase mixture coexists. Because of technical computing limitations we compute the gas spinodal only at temperatures close to the critical one, that is $9000 \mathrm{~K}$. Above the critical temperature $(9350 \mathrm{~K})$ the pressure decreases continuously with decreasing density, but does not show any minima or maxima. We obtain the critical point to be in the range $1.85-2.40 \mathrm{~g} / \mathrm{cm}^{3}$ and $9000-9350 \mathrm{~K}$ (black empty rectangle). The right inset shows comparisons of the critical point, between our estimate and the ones inferred from experiments (Fortov and Lomonosov, 2010; Medvedev, 2014). (For interpretation of the colors in the figure(s), the reader is referred to the web version of this article.)

at 130 GPa. Previous shock experiments (Chen and Ahrens, 1997) on face-centered cubic iron with an initial condition at $1570 \mathrm{~K}$ and 1 bar show that the Hugoniot intercepts the iron melting curve at $80 \mathrm{GPa}$. In the second case we consider the initial state at $40 \mathrm{GPa}$ and $4000 \mathrm{~K}$, which may be representative for the state of the core in Mars-size impactors (Canup, 2004b). At these conditions iron is already molten. Fig. 2(a) shows the computed Hugoniot EOS for these two cases. During shocks the temperature can easily reach thousands of degrees and the pressures hundreds of GPa. These would be typical conditions for the core state during the Giant Impact.

Then the core, as well as the rest of the protolunar disk or of the synestia will start to cool down and to depressurize along quasi-isentropic trajectories. The position of the iron CP that we find in our $a b$ initio molecular dynamics simulations is inside the outer surface of synestias (Lock et al., 2018). This implies that both the impactor's core and the proto-Earth's core might evolve at temperatures and pressures higher than those of the critical point throughout the giant impact and the condensation of the protolunar disk. Only iron ejected in the outermost parts of the disk, like the one originating from the impactor's core can reach low-enough pressures and temperatures to actually arrive at the conditions of the liquid-vapor dome. Indeed, the temperatures in these outer regions of the disk or of the synestia are low enough not to exceed the critical temperature and the pressures are below the liquid spinodal so that vaporization conditions can be attained.

The actual amount of vaporization possible after an impact depends on entropy. The entropy at the boiling point was estimated at $15.84 \mathrm{k}_{\mathrm{B}} /$ atom at $3100 \mathrm{~K}$ and 1 bar (Kraus et al., 2015). If the 


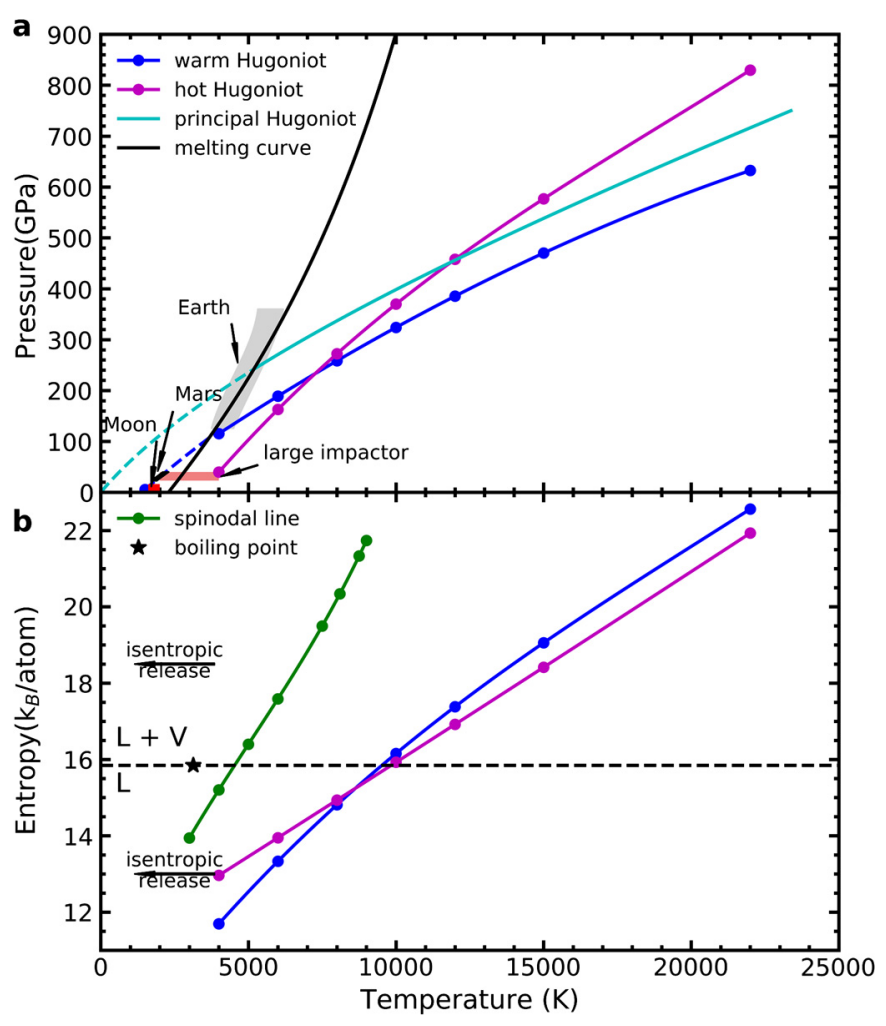

Fig. 2. Computed Hugoniot lines for iron starting from two realistic warm initial states. (a) Temperature - pressure plots for various impact scenarios. The principal Hugoniot (Sjostrom and Crockett, 2018) that starts at ambient conditions and the melting curve of iron (Bouchet et al., 2013) are shown for reference only. In general, impacts with Moon-sized impactors (Cuk and Stewart, 2012) yield hot final states, impacts with Mars-sized impactors (Canup, 2012) yield higher pressure final states. For the former case the warm initial state conditions are at $1 \mathrm{GPa}$ and 1500 $\mathrm{K}$, for the latter case the hot initial state is at $40 \mathrm{GPa}$ and $4000 \mathrm{~K}$. The Hugoniot lines cross because the gains in temperature and pressure is not linear with respect to changes in initial conditions. The shaded area represents estimated temperature gradients ranges in the metallic cores of the different objects involved in the impact (Antonangeli et al., 2015; Hirose et al., 2013; Stewart et al., 2007). (b) Computed entropy along the two Hugoniot lines. The star indicates the experimentally estimated entropy of boiling liquid iron at $3000 \mathrm{~K}$, marked also by the dashed line.

peak shock conditions during impact exceed this entropy value, then the onset of vaporization may take place and part of the shocked material can vaporize upon release and cooling (Ahrens and O'Keefe, 1972). We compute the entropies of the liquid along the two Hugoniot lines and at the spinodal points (Fig. 2(b) and Table 1) as a function of temperature from the vibrational spectra (Supplementary Material Fig. S1). For peak shock conditions at $15000 \mathrm{~K}$, we estimate that entropy can reach $19.1 \mathrm{k}_{\mathrm{B}}$ /atom and $18.6 \mathrm{k}_{\mathrm{B}}$ /atom along the warm and the hot Hugoniot curves respectively. At these conditions the entropy is high enough to result in partial vaporization of the iron. Our results show that above 7500 $\mathrm{K}$, the entropy along the warm Hugoniot is less than along the hot Hugoniot. The entropy difference between these two Hugoniot lines is relatively small $\left(0.5 \mathrm{k}_{\mathrm{B}}\right.$ /atom) in the $7500 \mathrm{~K}$ to 15000 $\mathrm{K}$ range. If we relate entropy to the peak shock pressure, based on the computed entropy along the warm Hugoniot line, we find that the shock pressure required to reach the onset of vaporization upon release and cooling is $312 \mathrm{GPa}$. This is less than previous estimates of $390 \mathrm{GPa}$ (Kraus et al., 2015). Along the hot Hugoniot, the onset vaporization pressure is $365 \mathrm{GPa}$. This is only slightly higher than that of warm Hugoniot.

The onset of core vaporization can easily be reached in case of impacts of small planetesimals, like the ones that might have occurred either during the first stages of formation of the solar system, or during the late veneer. As a model example we consider
Table 1

Temperature, density and entropy along the spinodal and two Hugoniot lines.

\begin{tabular}{|c|c|c|c|}
\hline Temperature (K) & Density $\left(\mathrm{g} / \mathrm{cm}^{3}\right)$ & Pressure (GPa) & Entropy $\left(\mathrm{k}_{\mathrm{B}} /\right.$ atom $)$ \\
\hline & \multicolumn{3}{|l|}{ spinodal } \\
\hline 3000 & 6.25 & -10.00 & 13.94 \\
\hline 4000 & 5.70 & -7.23 & 15.19 \\
\hline 5000 & 5.22 & -5.03 & 16.45 \\
\hline 6000 & 4.62 & -3.08 & 17.53 \\
\hline 7500 & 3.57 & -0.91 & 19.51 \\
\hline 8100 & 3.11 & -0.25 & 20.39 \\
\hline 8750 & 2.44 & 0.26 & 21.24 \\
\hline \multirow[t]{2}{*}{9000} & 2.40 & 0.42 & 21.79 \\
\hline & \multicolumn{3}{|c|}{ the warm Hugoniot } \\
\hline 4000 & 10.90 & 129.87 & 11.65 \\
\hline 6000 & 11.63 & 199.56 & 13.42 \\
\hline 8000 & 12.17 & 263.39 & 14.84 \\
\hline 10000 & 12.62 & 322.35 & 16.07 \\
\hline 12000 & 13.01 & 378.95 & 17.36 \\
\hline 15000 & 13.52 & 459.61 & 19.10 \\
\hline 22000 & $\begin{array}{l}14.47 \\
\text { the hot Hugonio }\end{array}$ & the hot Hugoniot & 22.56 \\
\hline 4000 & 8.85 & 40.00 & 13.18 \\
\hline 6000 & 11.05 & 161.62 & 13.75 \\
\hline 8000 & 12.30 & 273.67 & 14.79 \\
\hline 10000 & 13.14 & 369.79 & 15.90 \\
\hline 12000 & 13.81 & 457.36 & 16.94 \\
\hline 15000 & 14.60 & 577.05 & 18.60 \\
\hline 22000 & 15.98 & 829.76 & 21.87 \\
\hline
\end{tabular}

a differentiated planetesimal with a mantle made of enstatite and a core made of iron; we set the core-mantle boundary at $1 \mathrm{GPa}$ and $1500 \mathrm{~K}$ (Raymond et al., 2009). We approximate the shock wave as a planar wave (Melosh, 2011) traveling through the two layers. However, this yields a simplified estimate of the peak pressure and does not thoroughly describe the pressure distributions in these bodies. When the impact occurs, shock waves travel through the silicate layers of the two bodies. At the core-mantle boundary, because of the density contrast between silicates and iron, the shock wave is partly reflected, going backward into the mantle, and partly transmitted, going forward into the core. Assuming a steady shock in a model $\mathrm{MgSiO}_{3}$-based mantle (Militzer, 2013) and in the iron core, the impedance match method allows us to determine the properties of the reflected wave in the mantle and the transmitted wave in the core (Forbes, 2012). Fig. 3 illustrates the propagation of the shock wave according to this model through the planetesimal.

As the shock proceeds through the mantle of the impactor this can lead to partial fragmentation. During this process the mantle fragments and can detach from the core leaving behind bare fragments of shocked core. In the post-shocked state, fragments of planetesimal core without mantle confinement can undergo an isentropic release into vacuum. In this case, if the entropy reached during the shock is high enough, the core may partly vaporize; otherwise it will remain liquid and accrete to the impacted body, or escape gravitationally and eventually crystalize.

\subsection{Vaporization of small planetesimals}

For heads-on collisions of small planetesimals, the impact velocity required to onset vaporization is around $11.5 \mathrm{~km} / \mathrm{s}$. With an impact velocity around $13.5 \mathrm{~km} / \mathrm{s}$, peak pressure and temperature reach $450 \mathrm{GPa}$ and $15000 \mathrm{~K}$ (Fig. 4). At these conditions about $22 \%$ of iron would vaporize. In contrast, oblique impacts greatly reduce the peak pressure, due to a $\sin (\theta)$ factor where $\theta$ is the obliquity (Pierazzo and Melosh, 2000a). For the maximum frequency impact angles of $45^{\circ}$ (Pierazzo and Melosh, 2000b), the velocity threshold to onset vaporization increases to $15.3 \mathrm{~km} / \mathrm{s}$. With mean impact velocities at $14.5 \mathrm{~km} / \mathrm{s}$ and median impact angles at $40^{\circ}$, around $70 \%$ of the impacts of $\mathrm{N}$-body simulations (Raymond et al., 


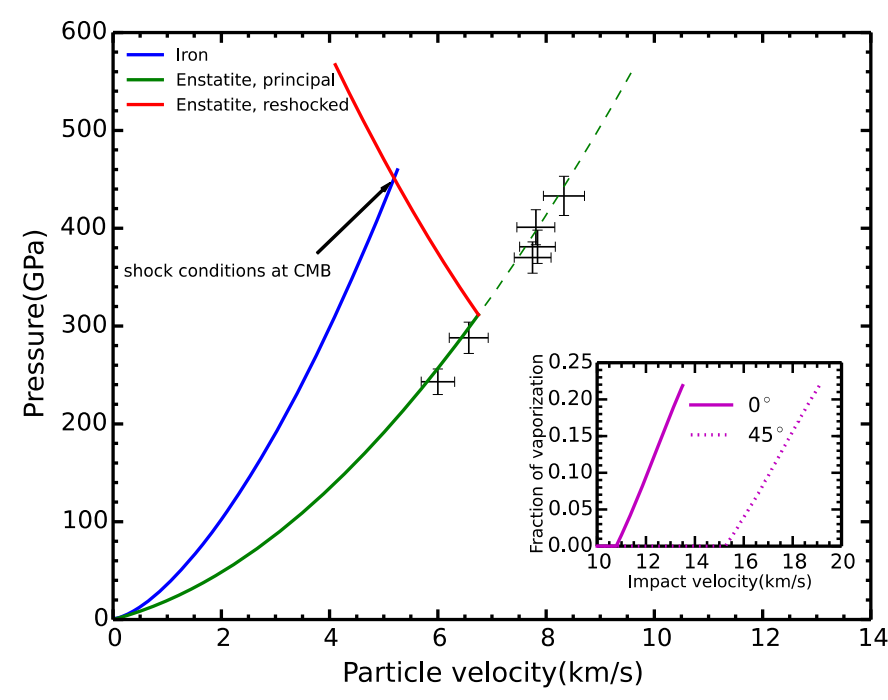

Fig. 3. Graphical representation of the impedance match analysis for the core of the small impactor. The shock wave generated at the moment of the impact travels through the impactor's mantle assumed to be homogeneous. The shock state at the core-mantle is given by the $\mathrm{MgSiO}_{3}$ principal Hugoniot (green line) estimated based on previous ab initio simulations (Militzer, 2013). More recent experimental Hugoniot data points are represented by crosses (Fratanduono et al., 2018). At the core-mantle boundary the shock wave splits in two opposite waves. The one traveling forward enters the core. The final state in the core is given by the intersection between the reshocked $\mathrm{MgSiO}_{3}$ Hugoniot (red line) and the iron Hugoniot (blue line) with an initial state at $1 \mathrm{GPa}$ and $1500 \mathrm{~K}$ (the warm Hugoniot in Fig. 2). The inset shows the fraction of iron that would vaporize from the corresponding impactor's core as a function of the impact velocity.

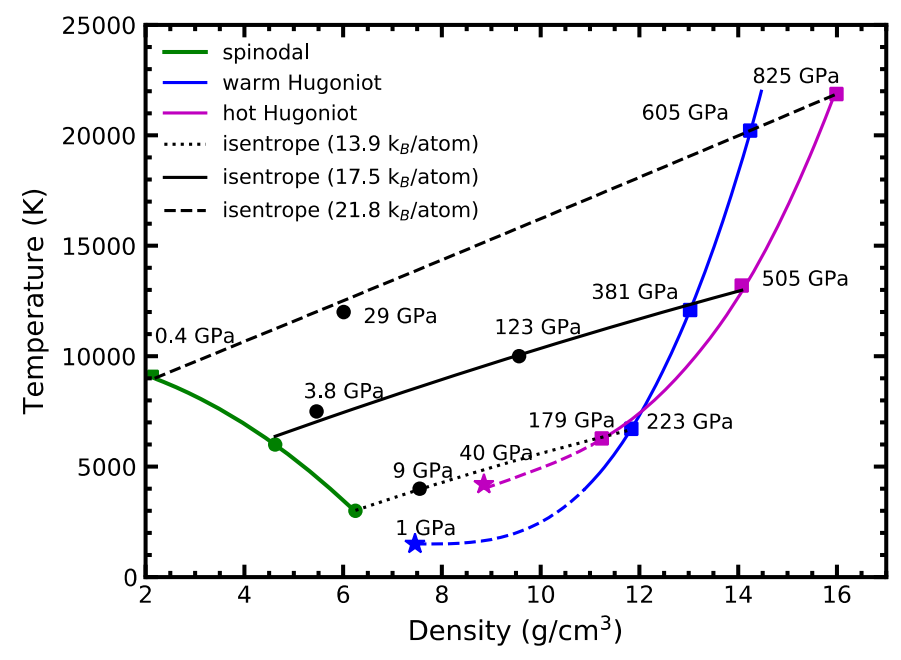

Fig. 4. Isentropic release of the iron core starting from various peak conditions attained during large impacts. The shocked material gains entropy that is conserved during the release. If this entropy is larger than that of the boiling point then partial vaporization may occur. We compute the entropy along the spinodal and the Hugoniot lines at different temperatures, which allows us to map the entropy increase during various impact scenarios. We find that the entropy of the critical point, at $21.8 \mathrm{k}_{\mathrm{B}} /$ atom, is reached for impacts with peak pressures of $605 \mathrm{GPa}$ in the case of small planetesimals or for peak pressures of $825 \mathrm{GPa}$ in the case of Mars-sized objects. Peak pressures of $312 \mathrm{GPa}$ are enough to provide entropy higher than the entropy at boiling of iron at $1 \mathrm{~atm}$, i.e. $15.84 \mathrm{k}_{\mathrm{B}}$ /atom (Kraus et al., 2015). These conditions can be easily exceeded during the Giant Impact between the proto-Earth and Theia, but could also be reached in almost half of the impacts with planetesimals during the late veneer. However, the liquid-vapor dome is reached only if the density, and hence the pressure, is allowed to decrease sufficiently. This can happen if the mantle is stripped away when fragments of the core are allowed to decompress without the mantle confinement.

2009) yield velocities larger than our threshold. This suggests that core vaporization is a common process during planetary formation. During the collision with the Earth's mantle, the core of the incom- ing planetesimal can be efficiently mixed into the molten silicate pond locally produced by the impact itself or into a pre-existing larger magma ocean. If such impacts happen after the Earth's core formation, this process would then increase the amount of highly siderophile elements that is seen today trapped and dispersed into the Earth's mantle.

\subsection{Vaporization during the Giant Impact}

In the case of the Giant Impact, the geometry effect plays an important role in controlling the shock peak conditions. As the validity of the impedance matching method is limited to the impacts where the lateral dimension of the impactor is small compared to the distance the shock wave has propagated (Melosh, 2011), it has only a limited applicability. However, the entropic and pressure criteria for vaporization still hold. For impacts with Mars-sized bodies, because of the hotter initial state of their cores, our simulations suggest a vaporization pressure of only $312 \mathrm{GPa}$. This is again smaller than previous estimations by Kraus et al. (2015) suggesting that even more iron will be vaporized than previously thought.

However, the amount of iron that can be vaporized depends also on the local pressure conditions as the process of the impact itself takes its due course. As the predicted pressure thresholds for vaporization can be easily reached, a large amount of iron receives enough entropy to vaporize. The entropy threshold can even be easier exceeded due to the entropy gain after the first and secondary shocks and the conversion of gravitational potential energy to internal energy (Carter et al., 2020; Nakajima and Stevenson, 2015). Once again during this process the confinement of core fragments by the surrounding mantle may prohibit the isentropic expansion and thus the vaporization. But if during the impact parts of the mantle are detached from fragments of the core (Nakajima and Stevenson, 2015) then during the isentropic release the pressure on those core fragments may drop below about 1 GPa (Fig. 3). These fragments will then undergo partial vaporization. Part of the vapor will remain in the outer part of the disk and eventually condense to form the Moon's core while the rest will fall into the central body and mix into the magma ocean.

Kendall and Melosh (2016) suggest fully mixing between impactor's core and proto-Earth Magma Ocean can be achieved for iron blobs that are less than $100 \mathrm{~km}$ across. Consequently, core fragmentation, promoted by partial vaporization during release, will enhance equilibrium and/or mixing between the impactor's core and the molten silicates, on a larger degree than the previous estimations of hydrodynamic simulations, which generally predicted the impactor's core directly merge into the Earth's core (Canup, 2012; Cuk and Stewart, 2012). Then the mixing process can easily explain the recent $\mathrm{W}$-isotope data, which require at least $30 \%$ core-mantle equilibration in the aftermath of the giant impact (Nimmo et al., 2010; Rudge et al., 2010; Touboul et al., 2015).

Stirring and disruption during the giant impact and inside the protolunar disk can bring large parts of the silicates mantle and of the impactor's core in contact with each other. As our results and previous work (Carter et al., 2020; Nakajima and Stevenson, 2015) show there is a large entropy gain during the impact, it is conceivable that the temperature rise due to this gain is enough to enhance the mixing of lithophile elements with iron metal, which subsequently increase the lithophile element content of the Earth's core. This would boost the mantle-core chemical exchange and equilibration from an early stage and provide the necessary initial state from which chemical unmixing can proceed to fuel the first stages of the dynamo, as was suggested in experiments recording the exsolution of various lithophile components from the liquid core (Badro et al., 2016; Hirose et al., 2017). 


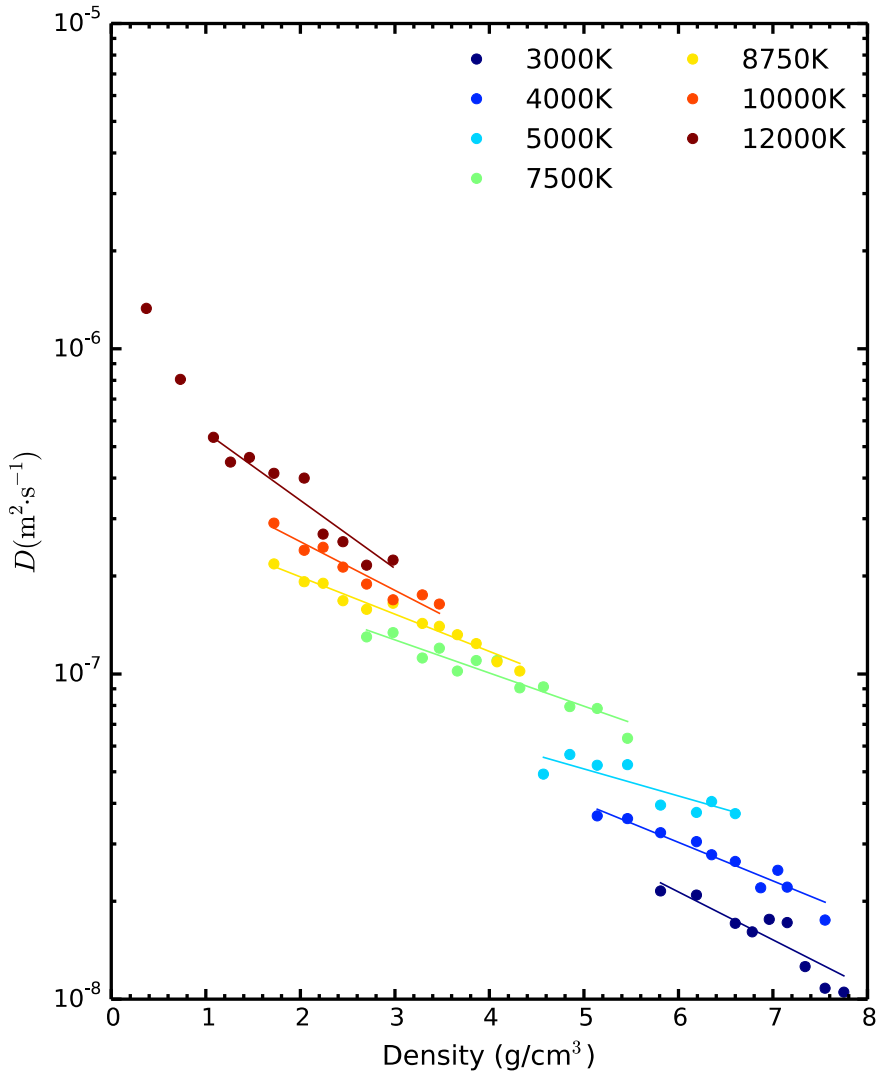

Fig. 5. The diffusion coefficients of the fluid iron as a function of density for various temperatures. These are obtained from the slope of the atomic mean-square displacements as a function of time (Supplementary Material Fig. S2).

\subsection{Transport properties of the various fluid phases of iron}

The partial core vaporization would also strongly affect the transport properties of the materials constituting the protolunar disk or the synestia. There are almost two orders of magnitude difference in the diffusion coefficients of liquid iron deep inside the disk and of the supercritical iron in the outer parts of the disk. This comes from a combination of temperature and density differences, which is directly translated into different mixing rates and different chemical and isotopic exchanges with the surrounding silicates.

The diffusivity increases by about two orders of magnitude from $1.05 \times 10^{-8} \mathrm{~m}^{2} \mathrm{~s}^{-1}$ at $7.75 \mathrm{~g} / \mathrm{cm}^{3}$ and $3000 \mathrm{~K}$ to $1.33 \times 10^{-6}$ $\mathrm{m}^{2} \mathrm{~s}^{-1}$ at $0.37 \mathrm{~g} / \mathrm{cm}^{3}$ and $12000 \mathrm{~K}$ (Fig. 5a). The general effect of decreasing the density or increasing the temperature is to enhance the atomic mobility. Below $12000 \mathrm{~K}$, the diffusivity - density relation can be described as a power law. However, at $12000 \mathrm{~K}$, there is a significant deviation from this trend, especially below 1.46 $\mathrm{g} / \mathrm{cm}^{3}$. With such high diffusion coefficients, our results suggest that chemical exchanges would be enhanced in the supercritical state.

\section{Conclusions}

We perform ab initio molecular dynamics to determine the position of the supercritical point of iron, and to characterize the fluid iron over a wide density and temperature range, with a special focus on the supercritical state. Based on our calculations, we predict the critical point of iron to be in the 9000-9350 K temperature range and $1.85-2.40 \mathrm{~g} / \mathrm{cm}^{3}$ density range, corresponding to a pressures range of $4-7$ kbars.
The determination of the Hugoniot equations of state and our estimations of the amounts of entropy gained during the Giant Impact show that the core of Theia underwent partial vaporization. Part of this vapor may have remained in the disk to eventually participate to the Moon's small core, another part may have fallen back to the proto-Earth. The presence of vapor considerably increases the mobility of iron, at the atomic level, thus enhances chemical mixing. Moreover, during the late veneer, a large fraction of the planetesimals' cores would undergo partial vaporization. This would help mixing the highly siderophile elements into magma ponds or oceans (Rubie et al., 2015).

Concerning Theia's core, its partial vaporization contributed to its fragmentation. This was not captured in the early numerical simulations of impacts (Canup, 2012; Cuk and Stewart, 2012), as their resolution was typically on the order of the $100 \mathrm{~km}$, suggesting more work should be dedicated to the resolution aspects. These simulations also do not model phase separation between liquid and vapor although they take into account explicitly the possible two-phase evolution of the iron part of the two bodies.

In general, our results are on the lower end of the range of thresholds for velocities obtained from ANEOS extrapolations (Pierazzo et al., 1997), which overestimate the onset pressure and thus limits the vapor production. But as ANEOS extrapolations are based on liquid and solid phases, without experimental or numerical data points on the vapor side of the dome, they can easily fail at high temperature and low densities. New impact simulations are clearly needed to understand the behavior of the core during giant impacts; the use of our ab initio results related to the position of the $\mathrm{CP}$ and the entropy during shock, together with better high-density EOS would definitely improve the reliability of such disk-scale simulations.

\section{Declaration of competing interest}

The authors declare that they have no known competing financial interests or personal relationships that could have appeared to influence the work reported in this paper.

\section{Acknowledgements}

The authors thank two anonymous reviewers whose comments greatly improved the manuscript. This research was supported by the European Research Council (ERC) under the European Union's Horizon 2020 research and innovation program (grant agreement no. 681818 IMPACT to RC) and under the Marie Skłodowska-Curie program (grant agreement no. 750901 ABISSE to FS). RC acknowledges support from the Research Council of Norway through its Centres of Excellence funding scheme, project number 223272. We acknowledge access to the GENCI supercomputers (Occigen, Ada, Jean-Zay, and Curie) through the stl2816 series of eDARI computing grants, and the TGCC supercomputers (Irene) through the PRACE grant RA4947.

\section{Appendix A. Supplementary material}

Supplementary material related to this article can be found online at https://doi.org/10.1016/j.epsl.2020.116463.

\section{References}

Ahrens, T.J., O'Keefe, J.D., 1972. Shock melting and vaporization of lunar rocks and minerals. Moon 4, 214-249. https://doi.org/10.1007/BF00562927.

Alfè, D., Gillan, M.J., Price, G.D., 1999. The melting curve of iron at the pressures of the Earth's core from ab initio calculations. Nature 401, 462-464. https:// doi.org/10.1038/46758.

Antonangeli, D., Morard, G., Schmerr, N.C., Komabayashi, T., Krisch, M., Fiquet, G., Fei, Y., 2015. Toward a mineral physics reference model for the Moon's core. Proc Natl. Acad. Sci. 112, 3916-3919. https://doi.org/10.1073/pnas.1417490112. 
Asphaug, E., 2014. Impact origin of the Moon? Annu. Rev. Earth Planet. Sci. 42, 551-578. https://doi.org/10.1146/annurev-earth-050212-124057.

Badro, J., Siebert, J., Nimmo, F., 2016. An early geodynamo driven by exsolution of mantle components from Earth's core. Nature 536, 326-328. https://doi.org/10. 1038/nature18594.

Blöchl, P.E., 1994. Projector augmented-wave method. Phys. Rev. B 50, 17953-17979. https://doi.org/10.1103/PhysRevB.50.17953.

Bouchet, J., Mazevet, S., Morard, G., Guyot, F., Musella, R., 2013. Ab initio equation of state of iron up to 1500 GPa. Phys. Rev. B 87. https://doi.org/10.1103/PhysRevB. 87.094102 .

Campbell, A.J., 2016. Phase diagrams and thermodynamics of core materials. In: Terasaki, H., Fischer, R.A. (Eds.), Geophysical Monograph Series. John Wiley \& Sons, Inc, Hoboken, NJ, pp. 191-199.

Canup, R.M., 2012. Forming a Moon with an Earth-like composition via a giant impact. Science 338, 1052-1055. https://doi.org/10.1126/science.1226073.

Canup, R.M., 2004a. Dynamics of lunar formation. Annu. Rev. Astron. Astrophys. 42, 441-475. https://doi.org/10.1146/annurev.astro.41.082201.113457.

Canup, R.M., 2004b. Simulations of a late lunar-forming impact. Icarus 168, 433-456. https://doi.org/10.1016/j.icarus.2003.09.028.

Canup, R.M., Righter, K. (Eds.), 2000. Origin of the Earth and Moon. The University of Arizona Space Science Series. University of Arizona Press/Lunar and Planetary Institute, Tucson/Houston.

Caracas, R., 2016. Crystal structures of core materials. In: Terasaki, H., Fischer, R.A. (Eds.), Geophysical Monograph Series. John Wiley \& Sons, Inc, Hoboken, NJ, pp. 55-68.

Carter, P.J., Lock, S.J., Stewart, S.T., 2020. The energy budgets of giant impacts. J. Geophys. Res., Planets 125. https://doi.org/10.1029/2019JE006042.

Chen, G.Q., Ahrens, T.J., 1997. High pressure and high temperature equation-of-state of gamma and liquid iron. MRS Proc. 499. https://doi.org/10.1557/PROC-499-41.

Cuk, M., Stewart, S.T., 2012. Making the Moon from a fast-spinning Earth: a gian impact followed by resonant despinning. Science 338, 1047-1052. https://doi. org/10.1126/science.1225542.

Desjarlais, M.P., 2013. First-principles calculation of entropy for liquid metals. Phys. Rev. E 88. https://doi.org/10.1103/PhysRevE.88.062145.

Forbes, J.W., 2012. Shock Wave Compression of Condensed Matter. Springer Berlin Heidelberg, Berlin, Heidelberg

Fortov, V.E., Lomonosov, I.V., 2010. Shock waves and equations of state of matter Shock Waves 20, 53-71. https://doi.org/10.1007/s00193-009-0224-8.

Fratanduono, D.E., Millot, M., Kraus, R.G., Spaulding, D.K., Collins, G.W., Celliers, P.M., Eggert, J.H., 2018. Thermodynamic properties of $\mathrm{MgSiO}_{3}$ at super-Earth mantle conditions. Phys. Rev. B 97. https://doi.org/10.1103/PhysRevB.97.214105.

Green, J.L., Durben, D.J., Wolf, G.H., Angell, C.A., 1990. Water and solutions at negative pressure: Raman spectroscopic study to -80 megapascals. Science 249 , 649-652. https://doi.org/10.1126/science.249.4969.649.

Grosse, A.V., Kirshenbaum, A.D., 1963. The densities of liquid iron and nickel and an estimate of their critical temperature. J. Inorg. Nucl. Chem. 25, 331-334. https:/ doi.org/10.1016/0022-1902(63)80181-5.

Hirose, K., Labrosse, S., Hernlund, J., 2013. Composition and state of the core Annu. Rev. Earth Planet. Sci. 41, 657-691. https://doi.org/10.1146/annurev-earth050212-124007.

Hirose, K., Morard, G., Sinmyo, R., Umemoto, K., Hernlund, J., Helffrich, G., Labrosse, S., 2017. Crystallization of silicon dioxide and compositional evolution of the Earth's core. Nature 543, 99-102. https://doi.org/10.1038/nature21367.

Hixson, R.S., Winkler, M.A., Hodgdon, M.L., 1990. Sound speed and thermophysica properties of liquid iron and nickel. Phys. Rev. B 42, 6485-6491. https://doi.org/ 10.1103/PhysRevB.42.6485.

Karthika, S., Radhakrishnan, T.K., Kalaichelvi, P., 2016. A review of classical and nonclassical nucleation theories. Cryst. Growth Des. 16, 6663-6681. https:// doi.org/10.1021/acs.cgd.6b00794.

Kendall, J.D., Melosh, H.J., 2016. Differentiated planetesimal impacts into a terrestrial magma ocean: fate of the iron core. Earth Planet. Sci. Lett. 448, 24-33. https:/ doi.org/10.1016/j.epsl.2016.05.012.

Kraus, R.G., Root, S., Lemke, R.W., Stewart, S.T., Jacobsen, S.B., Mattsson, T.R., 2015. Impact vaporization of planetesimal cores in the late stages of planet formation Nat. Geosci. 8, 269-272. https://doi.org/10.1038/ngeo2369.

Kresse, G., Furthmüller, J., 1996. Efficient iterative schemes for ab initio total-energy calculations using a plane-wave basis set. Phys. Rev. B 54, 11169-11186. https:// doi.org/10.1103/PhysRevB.54.11169.

Kresse, G., Joubert, D., 1999. From ultrasoft pseudopotentials to the projector augmented-wave method. Phys. Rev. B 59, 1758-1775. https://doi.org/10.1103/ PhysRevB.59.1758.

Lichtenstein, A.I., Katsnelson, M.I., Kotliar, G., 2001. Finite-temperature magnetism of transition metals: an ab initio dynamical mean-field theory. Phys. Rev. Lett. 87, 067205. https://doi.org/10.1103/PhysRevLett.87.067205.

Lin, S.-T., Blanco, M., Goddard, W.A., 2003. The two-phase model for calculating thermodynamic properties of liquids from molecular dynamics: validation for the phase diagram of Lennard-Jones fluids. J. Chem. Phys. 119, 11792-11805. https://doi.org/10.1063/1.1624057.

Lock, S.J., Stewart, S.T., Petaev, M.I., Leinhardt, Z., Mace, M.T., Jacobsen, S.B., Cuk, M., 2018. The origin of the Moon within a terrestrial synestia. J. Geophys. Res., Planets 123, 910-951. https://doi.org/10.1002/2017JE005333.

Marqués, M., González, L.E., González, D.J., 2015. Ab initio study of the structure and dynamics of bulk liquid Fe. Phys. Rev. B 92, 134203. https://doi.org/10.1103/ PhysRevB.92.134203

Medvedev, A.B., 2014. Wide-range multiphase equation of state for iron. Combust. Explos. Shock Waves 50, 582-598. https://doi.org/10.1134/S0010508214050141.

Melosh, H.J., 2011. Planetary Surface Processes. Cambridge University Press, Cambridge.

Militzer, B., 2013. Ab initio investigation of a possible liquid-liquid phase transition in $\mathrm{MgSiO}_{3}$ at megabar pressures. High Energy Density Phys. 9, 152-157. https:// doi.org/10.1016/j.hedp.2012.11.006.

Nakajima, M., Stevenson, D.J., 2015. Melting and mixing states of the Earth's mantle after the Moon-forming impact. Earth Planet. Sci. Lett. 427, 286-295. https:|| doi.org/10.1016/j.epsl.2015.06.023.

Nimmo, F., O’Brien, D.P., Kleine, T., 2010. Tungsten isotopic evolution during latestage accretion: constraints on Earth-Moon equilibration. Earth Planet. Sci. Lett. 292, 363-370. https://doi.org/10.1016/j.epsl.2010.02.003.

Nosé, S., 1984. A unified formulation of the constant temperature molecular dynamics methods. J. Chem. Phys. 81, 511-519. https://doi.org/10.1063/1.447334.

Perdew, J.P., Burke, K., Ernzerhof, M., 1996. Generalized gradient approximation made simple. Phys. Rev. Lett. 77, 3865-3868. https://doi.org/10.1103/ PhysRevLett.77.3865.

Pierazzo, E., Melosh, H.J., 2000a. Hydrocode modeling of oblique impacts: the fate of the projectile. Meteorit. Planet. Sci. 35, 117-130. https://doi.org/10.1111/j.19455100.2000.tb01979.x

Pierazzo, E., Melosh, H.J., 2000b. Understanding oblique impacts from experiments, observations, and modeling. Annu. Rev. Earth Planet. Sci. 28, 141-167. https:// doi.org/10.1146/annurev.earth.28.1.141.

Pierazzo, E., Vickery, A.M., Melosh, H.J., 1997. A reevaluation of impact melt production. Icarus 127, 408-423. https://doi.org/10.1006/icar.1997.5713.

Poole, P.H., Sciortino, F., Essmann, U., Stanley, H.E., 1992. Phase behaviour of metastable water. Nature 360, 324-328. https://doi.org/10.1038/360324a0.

Raymond, S.N., O’Brien, D.P., Morbidelli, A., Kaib, N.A., 2009. Building the terrestrial planets: constrained accretion in the inner solar system. Icarus 203, 644-662. https://doi.org/10.1016/j.icarus.2009.05.016.

Reed, M.S.C., Flurchick, K.M., 1994. Hybrid molecular dynamics: an approach to low density simulations. Comput. Phys. Commun. 81, 56-64. https://doi.org/10.1016/ 0010-4655(94)90110-4.

Rubie, D.C., Nimmo, F., Melosh, H.J., 2015. Formation of the Earth's core. In: Treatise on Geophysics. Elsevier, pp. 43-79.

Rudge, J.F., Kleine, T., Bourdon, B., 2010. Broad bounds on Earth's accretion and core formation constrained by geochemical models. Nat. Geosci. 3, 439-443. https:// doi.org/10.1038/ngeo872.

Sjostrom, T., Crockett, S., 2018. Quantum molecular dynamics of warm dense iron and a five-phase equation of state. Phys. Rev. E 97. https://doi.org/10.1103/ PhysRevE.97.053209.

Smith, R.F., Fratanduono, D.E., Braun, D.G., Duffy, T.S., Wicks, J.K., Celliers, P.M., Ali, S.J., Fernandez-Pañella, A., Kraus, R.G., Swift, D.C., Collins, G.W., Eggert, J.H., 2018. Equation of state of iron under core conditions of large rocky exoplanets. Nat. Astron. 2, 452-458. https://doi.org/10.1038/s41550-018-0437-9.

Speedy, R.J., 1982. Stability-limit conjecture. An interpretation of the properties of water. J. Phys. Chem. 86, 982-991. https://doi.org/10.1021/j100395a030.

Stewart, A.J., Schmidt, M.W., van Westrenen, W., Liebske, C., 2007. Mars: a new corecrystallization regime. Science 316, 1323-1325. https://doi.org/10.1126/science. 1140549.

Tateno, S., Hirose, K., Ohishi, Y., Tatsumi, Y., 2010. The structure of iron in Earth's inner core. Science 330, 359-361. https://doi.org/10.1126/science.1194662.

Touboul, M., Puchtel, I.S., Walker, R.J., 2015. Tungsten isotopic evidence for disproportional late accretion to the Earth and Moon. Nature 520, 530-533. https:// doi.org/10.1038/nature14355.

Vasisht, V.V., Saw, S., Sastry, S., 2011. Liquid-liquid critical point in supercooled silicon. Nat. Phys. 7, 549-553. https://doi.org/10.1038/nphys1993.

Waseda, Y., Suzuki, K., 1970. Atomic distribution and magnetic moment in liquid iron by neutron diffraction. Phys. Status Solidi B 39, 669-678. https://doi.org/10. 1002/pssb.19700390235.

Zhao, G., Yu, Y.J., Yan, J.L., Ding, M.C., Zhao, X.G., Wang, H.Y., 2016. Phase behavior of metastable liquid silicon at negative pressure: $a b$ initio molecular dynamics. Phys. Rev. B 93. https://doi.org/10.1103/PhysRevB.93.140203. 